\title{
Designing Hypergraph Layouts to GMPLS Routing Strategies ${ }^{\star}$
}

\author{
Jean-Claude Bermond ${ }^{1}$, David Coudert ${ }^{1}$, Joanna Moulierac ${ }^{1}$, \\ Stéphane Pérennes ${ }^{1}$, Ignasi Sau ${ }^{1,2}$, and Fernando Solano Donado ${ }^{3}$ \\ 1 Mascotte joint project, I3S(CNRS-UNS) INRIA, Sophia-Antipolis, France \\ 2 Graph Theory and Combinatorics Group at Applied Maths. IV Dept. of UPC, Barcelona, Spain \\ 3 Institute of Telecommunications, Warsaw University of Technology, Poland
}

\begin{abstract}
All-Optical Label Switching (AOLS) is a new technology that performs packet forwarding without any Optical-Electrical-Optical (OEO) conversions. In this paper, we study the problem of routing a set of requests in AOLS networks using GMPLS technology, with the aim of minimizing the number of labels required to ensure the forwarding. We first formalize the problem by associating to each routing strategy a logical hypergraph whose hyperarcs are dipaths of the physical graph, called tunnels in GMPLS terminology. Such a hypergraph is called a hypergraph layout, to which we assign a cost function given by its physical length plus the total number of hops traveled by the traffic. Minimizing the cost of the design of an AOLS network can then be expressed as finding a minimum cost hypergraph layout.

We prove hardness results for the problem, namely for general directed networks we prove that it is NPhard to find a $C \log n$-approximation, where $C$ is a a positive constant and $n$ is the number of nodes of the network. For symmetric directed networks, we prove that the problem is APX-hard. These hardness results hold even is the traffic instance is a partial broadcast. On the other hand, we provide $\operatorname{an} \mathcal{O}(\log n)$ approximation algorithm to the problem for a general symmetric network. Finally, we focus on the case where the physical network is a path, providing a polynomial-time dynamic programming algorithm for a bounded number of sources, thus extending the algorithm given in [2] for a single source.
\end{abstract}

\section{Introduction}

All-Optical Label Switching (AOLS) [11] is an approach to route packets transparently and all-optically, thus allowing a speed-up of the forwarding. This very promising technology for the future Internet applications also brings new constraints and new problems. Indeed, since the forwarding functions are implemented directly at the optical domain, a specific correlator (device) is needed for each optical label processed in the node. Therefore, it is of major importance to reduce the number of employed correlators in every node, implying a reduction in the number of labels (as referred in the rest of the paper) that are going to be used by the traffic. Due to its flexibility as a control plane and to the fact that it handles traffic forwarding, the Generic MultiProtocol Label Switching (GMPLS) is the most promising protocol to be applied in AOLS-driven networks.

In GMPLS, traffic is forwarded through logical connections called Label Switched Paths (LSPs). When GMPLS is used with packet-based network, packets are associated to LSPs by means of a label, or tag, placed on top of the header of the packet. In this way, routers - called Label Switched Routers (LSRs) - can distinguish and forward packets.

The GMPLS standards allow packets to carry a set of labels in their header, conforming a stack of labels. Even though a packet may contain more than one label, LSRs must only read the first (or top) label in the stack in order to take forwarding decisions. This helps to reduce both the number of labels that need to be maintained on the core LSRs and the complexity of managing data forwarding across the backbone.

Stacking labels and label processing, in general, are standardized by the following set of operations that an LSR can perform over a given stack of labels:

- SWAP: replace the label at the top by a new one,

- PUSH: replace the label at the top by a new one and then push one or more onto the stack, and

- POP: remove the label at top in the label stack.

* This work has been partly funded by the European project IST FET AEOLUS and the project "Optimization Models for NGI Core Network" (Polish Ministry of Science and Higher Education, grant N517 397334). 


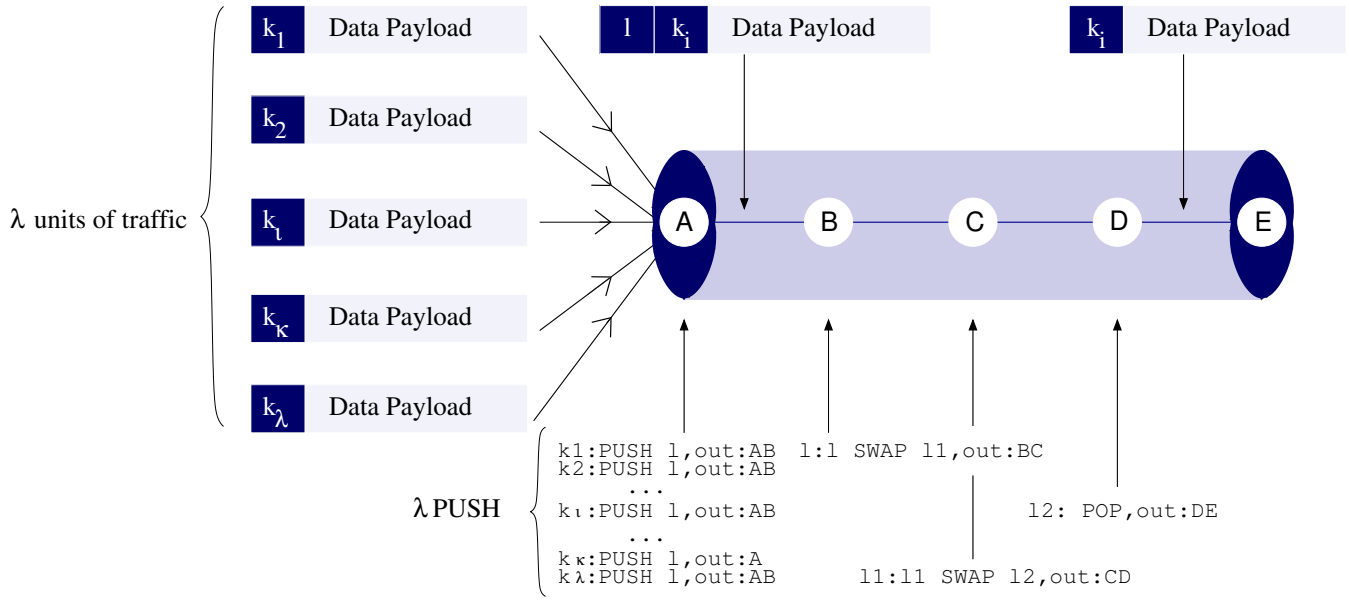

Fig. 1. GMPLS operations performed at the entrance and at the exit of a tunnel.

The labels stored in the forwarding table are significant only locally at the node and swapped all along the LSP (See Fig. 1).

Solutions deployed by GMPLS for reducing the number of labels are label merging $[5,13,15]$ (not discussed here) and label stacking [14,17]. With label stacking, when two or more LSPs follow the same set of links, they can be routed together "inside" a higher-level LSP, henceforth a tunnel. In order to setup a tunnel, multiple labels are placed in the packet's header.

Fig. 1 represents the general operations needed to configure a tunnel with the use of label stacking. At the entrance of the tunnel, $\lambda$ PUSH are performed in order to route the $\lambda$ units of traffic through the tunnel. Then, only one operation (either a SWAP or a POP at the end of the tunnel) is performed in all the nodes along the tunnel, regardless of $\lambda$. In this figure, a stack of size 2 is used to route the $\lambda$ LSPs in one tunnel from node $A$ to node $E$. The top label $l$ is swapped and replaced at each hop: by $l_{1}$ at node $B$, by $l_{2}$ at node $C$, and is finally popped at node $D$. The $\lambda$ units of traffic, at the exit of the tunnel at node $E$ can end or follow different paths according to their bottom label $k_{i}$, for all $i \in\{1,2, \ldots, w\}$ in the stack.

A consequence of the way in which the GMPLS operations can be configured at LSRs is the following: traffic can enter in any node of a tunnel but can exit in only one point, the last node of the tunnel. In other words, when some traffic is carried by a tunnel, it follows the tunnel until its end.

Since the number of labels used for GMPLS forwarding affects the cost of the AOLS architecture, in this paper we mainly focus on the minimization of the number of labels used. In our previous example, the total cost $c(T)$ of this tunnel $T$ from node $A$ to node $E$ in terms of number of labels is $c(T)=\lambda+\ell(T)-1$, where $\lambda$ is the number of units of traffic forwarded through this tunnel and $\ell(T)$ is its length in terms of number of hops (which is 4 on this example). We will formally define the cost function of the problem in Section 2.

Previous work and our contribution. The label minimization problem in GMPLS networks has been widely studied in the literature during the last few years [5,13-17]. All these articles focus mainly on proposing and analyzing heuristics to the problem, but there is a lack of theoretical results, like computational complexity or bounds on the approximation ratio of the proposed algorithms. For instance, in [16] the authors propose heuristics for routing a set of demands in AOLS networks when routers have limited number of available optical correlators. Very recently [2], the problem has been studied for the directed path from a more theoretical point of view. Namely, in [2] the authors present a polynomial-time optimal algorithm for the case when all traffic is issued from a single source and an $\mathcal{O}(\log n)$-approximation algorithm with arbitrary number of sources, where $n$ is the number of nodes of the network.

In this article we provide the first theoretical framework for the label minimization problem in general GMPLS networks. We translate the problem into finding a set of dipaths in a directed hypergraph. With this new formulation, it turns out that the problem is very similar to classical Virtual Path Layout (VPL) problems originating from ATM networks. We provide hardness results and approximation algorithms for the problem in general graphs. The approximation algorithms strongly rely on the already known algorithms for VPL problems. Finally, we focus on the path topology, extending the dynamic programming approach presented in [2] to any 
bounded number of sources. If there are $k$ sources, the main result is an optimal algorithm with running time $n^{\mathcal{O}(k)}$. That is, the problem is polynomial in the path for any fixed number of sources.

Organization of the paper. In Section 2 we formally state the problem in terms of hypergraph layout and fix the notation to be used throughout the article. In Section 3 we prove that for general directed networks it is NP-hard to find a $C \log n$-approximation, where $C$ is a a positive constant and $n$ is the number of nodes of the network. For symmetric directed networks, we prove that the problem is APX-hard, and therefore it does not accept a PTAS unless $\mathrm{P}=\mathrm{NP}$. In Section 4 we provide an approximation algorithm to the problem for symmetric directed graphs with an approximation ratio $\mathcal{O}(\log n)$, where $n$ is the number of nodes of the network. In Section 5 we focus on the directed path topology and present a dynamic programming approach solving the problem in polynomial time when the number of sources is fixed. Finally, Section 6 is devoted to conclusions and further research.

\section{GMPLS Logical Network Design as a Hypergraph Layout Problem}

The logical network design problem that we address can be roughly described as follows: we are given a digraph (directed graph) $G$ together with a set of traffic demands (or requests) between couples of vertices in $G$, and we must find a set of tunnels of minimum cost allowing to route all traffic requests. Note that usually communication networks are symmetric digraphs (i.e. when operators set a link on one direction, they also set the opposite link). So it is interesting to study the symmetric case, which turns out to be computationally easier than the general directed case. Let us now precise each one of the above terms.

A tunnel is simply a directed path (or dipath) in $G$, and due to the technological constraints discussed in Section 1, traffic can enter anywhere in the tunnel but must leave only at the end of the tunnel. To define the problem formally we need the following notation:

- $G=(V, E)$ is the underlying digraph (which can be symmetric or not).

- $|V|=n$, and vertices are numbered $1, \ldots, n$.

- $r_{i j}$ is the request from $i \in V$ to $j \in V$, with multiplicity $m_{i j} . R$ is the set of all requests.

- $P(G)$ is the set of all simple dipaths in $G$.

- $t$ stands for a tunnel, and $T$ is the set of tunnels, that is $t \in T \subseteq P(G)$.

- $\ell$ is a length function on the arcs, that is $\ell: E \rightarrow \mathbb{R}^{+}$.

- for a tunnel $t, \ell(t)=\sum_{e \in t} \ell(e)$ is its length and $w(t)$ is the amount of traffic it carries.

Note that a priori $w(t)$ depends on the routing policy. The cost of a tunnel $t$ is then $w(t)+(\ell(t)-1)$, and the cost of a set of tunnels $T$ is

$$
\sum_{t \in T}(w(t)+\ell(t)-1) .
$$

Each tunnel can be seen as a directed hyperarc on the vertex set of $G$. This observation naturally leads to the definition of a hypergraph layout.

Definition 1 (Hypergraph layout) Given a graph $G$ and a set $T \subseteq P(G), H(T)$ is the directed hypergraph with $V(H(T))=V(G)$, and where for each tunnel $t \in T \subseteq P(G)$ there is a directed hyperarc in $H(T)$ connecting any vertex of to the end of $t . H(T)$ is called a hypergraph layout.

Note that a hypergraph $H(T)$ defines a virtual topology on $G$. A hypergraph layout $H(T)$ is said to be feasible if for each request $r_{i j} \in R$ there exists a dipath in $H(T)$ from $i$ to $j$. The problem can then be simply expressed as finding a feasible hypergraph layout of minimum cost. Let us now rewrite the cost function of Equation (1).

Given a hypergraph layout $H(T)$, let $L\left(r_{i j}\right)$ be the number of hyperarcs that request $r_{i j}$ uses, and let $d_{H}(i, j)$ be the distance from vertex $i$ to vertex $j$ in $H(T)$. Then the term $\sum_{t \in T} w(t)$ of Equation (1) can be rewritten as $\sum_{r_{i j} \in R} L\left(r_{i j}\right) \cdot m_{i j}$ and, since $L\left(r_{i j}\right) \geq d_{H}(i, j)$, we conclude that in an optimal solution the routing necessarily uses shortest dipaths in the hypergraph layout. It follows that the cost function of Equation (1) can be rewritten w.l.o.g. as

$$
\sum_{t \in T}(\ell(t)-1)+\sum_{r_{i j} \in R} d_{H}(i, j) m_{i j}
$$


The cost of a solution is of bicriteria nature. The first part is the cost of the hypergraph structure; we call it the total length of the layout. The second part is the total distance that the traffic travels in the hypergraph; we call it the total hop count. Both cost function parts are very much conflicting. On the one hand, to minimize the hop count, it is enough to take a shortest tunnel connecting any source to any destination. On the other hand, to minimize the total length of the layout, it is enough to use a minimum arc-weighted connected hypergraph $H$ such that for each request $r_{i j} \in R$, vertices $i$ and $j$ lie on the same connected component of $H$. Summarizing, the problem can be stated as follows.

Minimum Cost Hypergraph Layout: Given a digraph $G$ with a length function and a set $R$ of traffic requests, find a feasible hypergraph layout of minimum cost, where the cost of a hypergraph layout is defined as in Equation (2).

If $G$ is a symmetric digraph, the problem is denoted Minimum Cost Symmetric Hypergraph Layout. It makes sense also to consider the decision version in which we are also given two positive integers $C_{L}, C_{H}$ and the objective is to decide whether there exists a layout with total length less than $C_{L}$ and total hop count less than $C_{H}$.

We note that the cost function of Equation (2) can be naturally generalized to

$$
\alpha \cdot \sum_{t \in T} c(t)+\beta \cdot \sum_{r_{i j} \in R} d_{H}(i, j) m_{i j},
$$

where $\alpha$ and $\beta$ are positive constants and $c(t)$ is a general cost function $c: P(G) \rightarrow \mathbb{R}^{+}$. The cost function of Equation (2) corresponds to $c(t)=\ell(t)-1$.

Relation with VPL problems. This layout design problem defined above is quite similar to well studied VPL problems in ATM networks, in which one imposes a constraint on the logical structure and then wishes to minimize either the maximum distance [3] or the average distance [8] traveled by the traffic. Concerning hardness and approximation, we shall see in the sequel of the article that the problem we study inherits most of the characteristics of the classical VPL problems studied since the 80 's. It is not surprising that, even if new technologies like GMPLS are proposed to cope with the increasing bandwidth of communication networks, the computational complexity of the problems associated to these technologies remains essentially the same.

Nevertheless, there are two crucial differences between the GMPLS problem that we study and the classical VPL version of ATM networks. Indeed, we have seen that the GMPLS logical network design problem can be translated into finding a set of dipaths in a directed hypergraph, whereas the existing models for VPL problems deal with digraphs without multiple arcs. This feature will be exploited in the dynamic programming approach for the path presented in Section 5. The second difference is that the cost function we consider takes into account the sum of the length and the hop count costs, whereas usually in VPL problems the aim is to minimize the

maximum value of either the length or the hop count in the network. Finally, it is important to note that, if there is a single source in the the GMPLS version (or, more generally, if the traffic instance is such that in an optimal solution each hyperarc has exactly 2 vertices), then the problem is basically equivalent to a classical VPL problem.

\section{Hardness Results}

In this section we give hardness results for the Minimum Cost Hypergraph Layout problem. We distinguish two cases according to whether the underlying network is symmetric or not. We focus on those cases in Sections 3.1 and 3.2 .

\subsection{General case}

Theorem 1 The Minimum Cost Hypergraph Layout problem cannot be approximated within a factor $C \log n$ for some constant $C>0$, even if the instance is a partial broadcast, unless $\mathrm{P}=\mathrm{NP}$. 


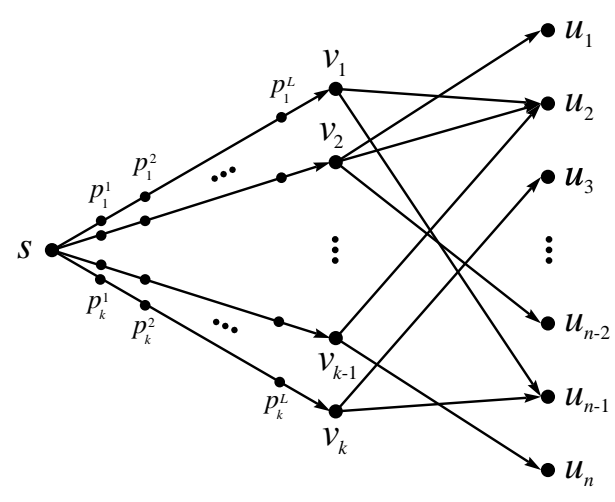

Fig. 2. Reduction in the proof of Theorem 1.

Proof: The reduction is from Minimum Set Cover ${ }^{4}$. Raz and Safra [12] proved that Minimum Set Cover is not approximable within a factor $C \log n$, for some constant $C>0$, unless $\mathrm{P}=\mathrm{NP}$. To a SET Cover instance with sets $S_{1}, S_{2}, \ldots, S_{k}$, with $S_{i} \subseteq\left\{a_{1}, a_{2}, \ldots, a_{n}\right\}$, we associate the following graph:

- We start with a distinguished node $s$.

- For each set $S_{i}$ we introduce a node $v_{i}$ and a directed path of length $L+1$ ( $L$ is a constant to be specified later) from $s$ to $v_{i}$ through $L$ new vertices $p_{i}^{1}, p_{i}^{2}, \ldots, p_{i}^{L}$.

- For each element $a_{j}$ we introduce a vertex $u_{j}$ and, for each vertex $v_{i}$ we add the $\operatorname{arcs}\left(v_{i}, u_{j}\right)$ if $a_{j} \in S_{i}$.

- The requests are from $s$ to $u_{j}$, for $i=1, \ldots, n$.

This construction is illustrated in Fig. 2. Let $O P T$ be the optimal cost to the Minimum Cost HypergraPH LAyOut instance, and let $O P T_{V C}$ be the optimal cost to the Minimum VerTEX Cover instance.

Note that any cover defined by $I \subseteq\{1,2, \ldots k\}$ induces a solution of DireCted Hypergraph Layout obtained as follows: we use a tunnel of cost $L$ connecting node $s$ to each node $v_{i}, i \in I$ corresponding to a set taken in the cover. Then we connect each node $v_{i}, i \in I$ to the vertices $u_{j}, j \in S_{i}$. Finally, if a node $u_{j}$ has more than one incoming tunnel (which means that $a_{j}$ is covered more than once), we remove extra ones. A solution induced by an optimal cover has length cost $L \cdot O P T_{V C}$, and the hop count cost is $2 n$, so $O P T \leq L \cdot O P T_{V C}+2 n$.

Conversely, given a layout, the dipaths from $s$ to $v_{i}$ used by some tunnel must induce a cover, so $O P T \geq$ $L \cdot O P T_{V C}+n$. Putting all together,

$$
L \cdot O P T_{V C}+n \leq O P T \leq L \cdot O P T_{V C}+2 n .
$$

By choosing $L$ to be large enough, the gap for the Minimum Cost Hypergraph Layout problem can be made as large as in Minimum Set Cover. Since, unless P = NP, approximating Minimum Set Cover within a factor $C \log n$ for some constant $C>0$ is NP-hard [12], our result follows.

\section{$3.2 \quad$ Symmetric case}

When the input graph $G$ is symmetric, we can consider $G$ as an undirected where the edge $\{i, j\}$ corresponds to the two $\operatorname{arcs}(i, j)$ and $(j, i)$.

Theorem 2 The Minimum Cost Symmetric Hypergraph Layout problem is APX-hard even if the instance is a partial broadcast. Therefore, it does not accept a PTAS unless $\mathrm{P}=\mathrm{NP}$.

Proof: The reduction is from Minimum Steiner Tree ${ }^{5}$, which is know to be APX-hard [4], hence it does not accept a PTAS unless $\mathrm{P}=\mathrm{NP}$.

Given an instance $(G=(V, E), S \subseteq V)$ of Minimum Steiner Tree problem on $n$ vertices, we build an instance of Minimum Cost Symmetric Hypergraph Layout problem by subdividing $\Omega\left(n^{2} \cdot \sum_{r_{i j} \in R} m_{i j}\right)$

\footnotetext{
${ }^{4}$ Given a finite set $\mathcal{S}$ and a collection $\mathcal{C}$ of subsets of $\mathcal{S}$, the aim is to find a subcollection $\mathcal{C}^{\prime}$ of $\mathcal{C}$ of minimum cardinality that covers all the elements of $\mathcal{S}$.

${ }^{5}$ Given an edge-weighted graph $G=(V, E)$ and a subset $S \subseteq V$, find a connected subgraph with minimum edge-weight containing all the vertices in $S$. We can assume, by subdividing edges, that all edge-weights equal 1.
} 
times each edge of $G$ and considering as request set a partial broadcast from any vertex in $S$ to all the others vertices in $S$. Note that subdividing edges is equivalent to setting $\alpha>>\beta$ in the cost function of Equation (3). In other words, the total hop count is negligible compared to the total length of the layout. It is then clear than any optimal solution to the Minimum Cost Symmetric Hypergraph Layout corresponds to a minimum cost Steiner tree in $G$ spanning all the elements in $S$. Let $O P T$ be the optimal cost to the Minimum Cost Symmetric Hypergraph Layout instance, and let $O P T_{S T}$ be the optimal cost to the Minimum Steiner TreE instance. Let $M$ be the number of times we have subdivided the edges of $G$. Summarizing,

$$
O P T=M \cdot O P T_{S T}+o\left(M \cdot O P T_{S T}\right) .
$$

The existence of a PTAS for Minimum Cost Symmetric Hypergraph Layout would yield a PTAS for Minimum Steiner Tree, which is impossible unless $\mathrm{P}=\mathrm{NP}$.

\section{Approximation Algorithms}

In this section we provide approximation algorithms for Minimum Cost HyPERGRAPH LAYOUT problem. Unless said otherwise, we focus on the symmetric version, for which the description of the algorithms is easier, although the main ideas could be adapted to the general version with slight modifications. For the sake of presentation, we describe our algorithms when the network is a path, a tree, and a general graph in Sections 4.1, 4.2, and 4.3, respectively. The approximation algorithm for the directed path network appeared also in [2], we include it here for the sake of completeness.

\subsection{Case of the path}

First assume that the instance is a weighted all-to-all (i.e., there is a traffic request between each couple of nodes), and that $n$ is a power of two (otherwise, just add dummy vertices). Then one simply uses the following binary layout: We connect node 1 to node $n / 2$, node $n / 2$ to node $n$, and we use recursively the binary layout for $n / 2$ on the subdipaths $[1, n / 2]$ and $[n / 2, n]$. It is clear that any traffic request can be routed in this layout with at most $\log n$ hops, and that the total length of this layout is bounded above by $\log n \cdot \ell([1, n])$, where $\ell([1, n])$ denotes the length of the tunnel going from node 1 to node $n$. Therefore the cost of this layout is $\log n \cdot \sum_{r_{i j} \in R} m_{i j}+\log n \cdot \ell([1, n])$. Since any layout costs at least $\sum_{d \in D} m_{i j}+\ell([1, n])$, this provides a $\log n-$ approximation in the all-to-all case.

Now, for a general traffic pattern, it is not always the case that $\ell([1, n])$ is a lower bound on the total length of the layout. We define the span of an instance as the minimum set of arcs such that any request can be routed using only those arcs. Note that the span is indeed a set of intervals such that any traffic request is routed within one of these intervals. Let $\ell_{0}$ denote the length of the span. Then any layout costs at least $\sum_{r_{i j} \in R} m_{i j}+\ell_{0}$, and using the binary layout on each interval of the span we can define a layout with total length $\log n \cdot \ell_{0}$ and total hop count $\log n \cdot \sum_{r_{i j} \in R} m_{i j}$. Summarizing,

Proposition 1. When the network is a path, there exists a polynomial-time approximation algorithm for MiNIMUM COST HyPERgRAPH LAYOUT problem with an approximation ratio $\mathcal{O}(\log n)$.

\subsection{Case of the tree}

In [3] the authors studied the design of virtual layouts in ATM networks. Their model deals with point-to-point connections in the virtual graph, whereas in Minimum Cost Hypergraph LAYout problem, a tunnel can carry more than one request. Nevertheless, we can use the results of [3] to obtain good approximation algorithms. Namely, we are interested in the following result which establishes the trade-off between the maximum load $c$ and the diameter of a virtual layout allowing to route an all-to-all traffic in a general tree.

Theorem 1 (Bermond et al. [3]). In a general tree on $n$ nodes with all-to-all traffic, for each value of $c \in\{1, \ldots, n\}$ there exists a virtual layout allowing to route all traffic with diameter at most $10 c \cdot n^{\frac{1}{2 c-1}}$ and load at most c. In addition, such a layout can be constructed in polynomial time. 
In particular, if we set $c=\frac{\log n+1}{2}$, Theorem 1 implies that we can find in polynomial time a layout with load $\mathcal{O}(\log n)$ and diameter at most $(5 \log n+5) \cdot n^{\frac{1}{\log n}}=5 \log n+5=\mathcal{O}(\log n)$.

Suppose first that the instance of Minimum Cost HyPERgRAPH LAYOut problem is a weighted all-to-all traffic. It is clear that each arc must be used by some tunnel, hence $n-1$ is a lower bound on the total length of any layout. On the other hand, the hop count is at least $\sum_{r_{i j} \in R} m_{i j}$. In the layout described above, each arc is used at most $\frac{\log n+1}{2}$ times, and therefore the total length of this layout is $\mathcal{O}(n \log n)$. Since the diameter is also $\mathcal{O}(\log n)$, the total hop count is $\mathcal{O}\left(\log n \cdot \sum_{r_{i j} \in R} m_{i j}\right)$, yielding an $\mathcal{O}(\log n)$-approximation.

If the instance is not all-to-all, we repeat the argument of the span discussed in Section 4.1, obtaining again an $\mathcal{O}(\log n)$-approximation. Summarizing,

Proposition 2. When the network is a tree, there exists a polynomial-time approximation algorithm for MiNIMUM COST HYPERGRAPH LAYOUT problem with an approximation ratio $\mathcal{O}(\log n)$.

\subsection{General network}

In the Minimum Generalized Steiner Network problem, we are given a graph $G=(V, E)$, a weight function $w: E \rightarrow \mathbb{N}$, a capacity function $c: E \rightarrow \mathbb{N}$, and a requirement function $r: V \times V \rightarrow \mathbb{N}$. The objective is to find a Steiner network over $G$ that satisfies all the requirements and obeys all the capacities, i.e., a function $f: E \rightarrow \mathbb{N}$ such that, for each edge $e, f(e) \leq c(e)$ and, for any pair of nodes $i$ and $j$, the number of edge disjoint paths between $i$ and $j$ is at least $r(i, j)$, where for each edge $e, f(e)$ copies of $e$ are available. We want to minimize the cost of the network, i.e., $\sum_{e \in E} w(e) f(e)$. The problem is approximable within $\mathcal{O}\left(\log r_{\max }\right)$, where $r_{\max }$ is the maximum requirement [9], and within a constant factor 2 when all the requirements are equal [10]. The directed version of the problem is approximable within $\mathcal{O}\left(n^{2 / 3} \log ^{1 / 3} n\right)$ [6].

Given an instance of Minimum Cost HyPERgraph LAyout in a general network, consider the associated Minimum Generalized Steiner Network problem where all the requirements are equal to 1 and where the edge capacities are set to $+\infty$. Let $H$ be an optimal solution to this Minimum Generalized Steiner NETwORK instance (note that $H$ may be disconnected). The following easy observation will be useful: since $H$ is the smallest subgraph of $G$ such that any couple source-destination lies on the same connected component, in any solution to the Minimum Cost HyPERgRaph Layout problem, the number of arcs that are used by at least one tunnel is at least $|E(H)|$. Using the algorithm of [10], we can find in polynomial time a Steiner network $H^{\prime}$ with $\left|E\left(H^{\prime}\right)\right| \leq 2|E(H)|$. Since the edge capacities are set to $\infty$, we can assume that such a Steiner network is a forest. The layout is then obtained by applying the algorithm described in Section 4.2 to each connected component of $H^{\prime}$.

It is clear that the hop count of this layout is at most $\mathcal{O}(\log n)$ times the lower bound $\sum_{r_{i j} \in R} m_{i j}$. On the other hand, the total length of this layout is $\mathcal{O}\left(\log n \cdot\left|E\left(H^{\prime}\right)\right|\right)=\mathcal{O}(\log n \cdot|E(H)|)$. Since the total length of any layout is lower-bounded by $|E(H)|$, the $\mathcal{O}(\log n)$-approximation follows. Summarizing,

Theorem 2. In a general network, there exists a polynomial-time approximation algorithm for MinIMUM CoST HYPERGRAPH LAYOUT problem with an approximation ratio $\mathcal{O}(\log n)$.

\subsection{Toward constant approximation in the single source case}

As already mentioned in Section 2, in the single source case our problem consists in finding a virtual layout with at most $\bar{h}$ hops and using a forest with length at most $L$. Note that we can consider two basic versions of the virtual network problem: we may use as first constraint either the maximal load of an edge, $L_{\max }$, or the average load, $\bar{L}$, and as second constraint the average hop count or the maximum hop count. Moreover, in each case one may either minimize the sum or just decide the feasibility of both constraints. Last, when studying approximated solutions, one may either relax both constraints or only one.

Minimizing the maximum load under a bound on the maximum hop count is rather easy (it is basically a classical VPL problem), and in bounded degree networks this load is of order $T^{1 / k}$ where $T$ is the amount of traffic. This bound can be obtained by sampling any given spanning tree.

On the other hand, when considering the average load of the edges, the problem gets more interesting since it is related to the $k$-centers problem. Since any tunnel can be assumed to be a shortest path, we can work in $K_{n}$ with an arbitrary metric. Since for uniform instance on most networks the average hop count will be, up to a constant, equal to the maximal number of hops, one may look at the bounded hops variant. When $h_{\max }=2$ 
our problem is indeed the undirected facility location [7] (by considering each node a facility with cost equal to its distance to the closest source, and by taking the distance from a facility to a node to be the distance in the graph. In [1] Kantor and Peleg studied the $k$-hops version under the name of hierarchical facility location, and gave a bounded approximation with ratio $O\left(2^{\theta(k)}\right)$. For example on a $n \times n$ grid with average hop count $<3$, we must have most of the $n^{2}$ nodes at distance 2 , and one easily proves that the cost will be of order $\min _{k}\left\{n k^{2}+k^{2}(n / k)^{3}\right\}=n^{7 / 3}$, and solutions for the average number of hop constraint do not differ significantly from the hierarchical facility location. So a minimum bounded depth spanning tree (equiv. depth $k$ hierarchical facility location) provides bounded approximation whenever $h_{\max }$ and $\bar{h}$ do not differ. The case in which a few nodes are kept far away to avoid dramatic increase in the layout structure cost remains to be investigated. To the best of our knowledge there do not exist results in the case of average number yet.

\section{The Hypergraph Layout Problem on the Path}

In this section we focus on the case when the underlying digraph is a directed path (Nodes are numbered from left to right $1, \ldots, n)$. Our approach consists in a dynamic programming algorithm that computes partial solutions induced on subdipaths of the original path. We denote by $[i, j]$ the subpath from node $i$ to node $j$.

Loosely speaking, we use the following dynamic program: we consider a cut vertex $i$ and we look at a local solution induced on the subpath $[1, i]$. That is, the tunnels and traffic located on $[1, i]$. The cost of a local solution is defined as the sum of the local tunnels cost plus the hop counts sum taken on the local traffic.

We introduce then node $i+1$ and the potential tunnels finishing at it. In order to update the local solution cost, it is necessary to have enough information to compute the hop counts once this tunnel is introduced in the solution. So for each source $s \in S$ and vertex $x$, we introduce $h(s, x)$ defined as the hop count from $s$ to $x$. Each vertex is then characterized by a hop count vector $h(x)$ whose dimension is the number of sources. A partial solution is then fully encoded by its local cost and the hop counts of all its nodes. It follows from the above discussion that we can encode a partial solution by giving, for each of its hop count vectors, the rightmost node associated to that vector. If we denote by $h$ a bound on the hop count (at most $n$ ) and by $c$ a bound (at most $n$ ) on the tunnel cost, we have $\left(c^{k}\right)^{h^{k}}=c^{k h^{k}}$ such possible table entries.

By making an error of $\varepsilon$ on the two costs (length and hops), we can encode the logarithm in base $1+\varepsilon$ of those quantities, which lead to tables of size $\Theta\left((\log n)^{k\left(\log n\left(^{k}\right.\right.}\right)$. Note that this running time is already subexponential, so the problem is unlikely to be NP-hard to approximate within a constant factor when the number of sources is bounded (because it is widely assumed that algorithms solving 3-SAT require $2^{\Theta(n)}$ time). We shall see now how to improve this first naïve dynamic program.

We proceed now to give all the details for one and two sources, that suffice to get the intuition for an arbitrary number $k$ of sources.

\subsection{Case of a single source and the non crossing property}

We summarize the algorithm that appeared first in [2] (Gerstel et al. used a similar approach in ??). In the case of a single source, it is not difficult to see that the tunnel structure is non crossing, i.e. two tunnels can only intersect in an optimal solution if one is strictly inside the other [2]. Since the path is directed, we assume w.l.o.g. that the source is located in the leftmost node of the path. This leads to the following approach: we consider the rightmost tunnel originating from the source and assume it ends at node $i$. Clearly, any tunnel starting in $[1, i-1]$ and ending in $[i, n]$ can be replaced with a tunnel starting at $i$, since this new tunnel may only decrease the hop count and the length ${ }^{6}$.

This approach allows us to compute the optimal for a path with $n$ vertices inductively. We denote by $C[i, j]$ the minimum cost for the requests destined to the subdipath $[i, j]$, in which the source is replaced by node $i$. Then for $2 \leq i \leq n$,

$$
C[1, i]=\min _{k<i}\left\{C[1, k-1]+\left(\sum_{e \in E([1, k])} \ell(e)-1\right)+\sum_{j=k}^{i} m_{1 j}+C[k, i]\right\} .
$$

\footnotetext{
${ }^{6}$ this fact holds for any increasing cost function $c(t)$ in Equation (3).
} 


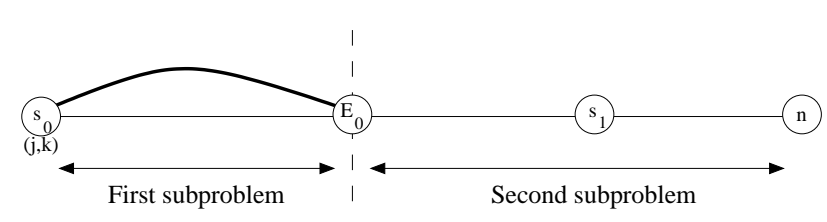

(a)

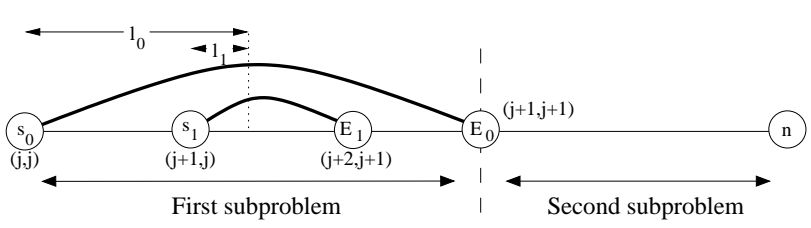

(b)

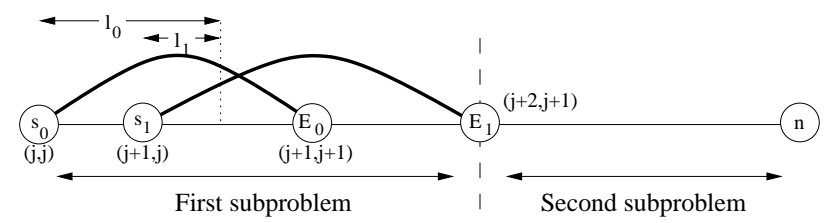

(c)

Fig. 3. Dynamic programming with two sources: cases (1), (2), and (3), respectively.

Note that $C[1, n]$ is the optimal cost of the original problem, and it can be computed in time $\mathcal{O}\left(n^{3}\right)[2]$. In the particular case of the uniform broadcast (that is, $m_{i j}=1$ for $i=1$ and $2 \leq j \leq n$, and $m_{i j}=0$ otherwise) and with a unitary length function on the edges, a closed formula was given in [2].

\subsection{Case of two sources}

We use a dynamic program similar to the one used for the single source case, but slightly more complicated. Let $s_{0}$ be the leftmost source and let $s_{1}$ be the other. In order to solve the problem, we introduce an auxiliary problem with pseudo-sources. A pseudo-source $s$ is denoted by a triple $\left(h_{0}, h_{1}, l\right)$, where $l$ is the distance from $s$ to the subdipath that lies on the right of the rightmost pseudo-source, and where $h_{i}$ indicates that from $s$ one can reach $s_{i}$ in $h_{i}$ hops, $i=0,1$. In the induction of the dynamic program the following auxiliary problem will appear:

- The traffic is restricted to an interval $[u, v]$, where either $u$ or $v$ is an end of the original dipath.

- There are one or two pseudo-sources located to the left of $[u, v]$.

- If there are two pseudo-sources, they are labeled $\left(j, j, l_{0}\right)$. and $\left(j+1, j, l_{1}\right)$, and we denote the corresponding problem $P\left((j, j),(j+1, j), l_{0}, l_{1},[u, v]\right)$

- If there a single pseudo-source, it is labeled $(j, k)$, and we denote the problem $P((j, k),[u, v])$.

In both cases we denote by $O P T$ the cost of an optimal solution. Note that $P((0,0),[u, v])$ is indeed a single source problem in which a unique source replaces both $s_{0}$ and $s_{1}$. Moreover, $P((j, k),[u, v])$ is equivalent to a single source problem, since $\left.O P T(P((j, k),[u, v]))=\operatorname{OPT}(P(0,0,[u, v]))+\sum_{x \in[u, v]}\left(j \cdot m_{s_{0} x}+k \cdot m_{s_{1} x}\right)\right)$. We now relate the two sources problem to the auxiliary problem. Consider the rightmost tunnel having $s_{i}$ as head and denote by $E_{i}$ its end node, $i=0,1$. We compute an optimal solution conditioned the values $E_{i}, i=0,1$. There are three cases to consider, as it is depicted in Fig. 3.

(1) $E_{0}$ is left to $s_{1}$. Then on the subpath $\left[E_{0}, n\right]$ we pick an optimal solution with a slightly modified instance: we leave traffic requests toward $s_{1}$ unchanged and we replace the source $s_{0}$ by a pseudo-source at $E_{0}$ with hop count 1 . On the subpath $\left[s_{0}, E_{0}-1\right]$ we use an optimal solution (note that in this subproblem there is only one source).

(2) $E_{0}$ is on the right of both $s_{1}$ and $E_{1}$. Then $E_{0}$ is at distance 1 from both sources and therefore any tunnel entering $\left[E_{0}+1, n\right]$ can be assumed to start at $E_{0}$. So the optimal solution is then obtained by using $\operatorname{OPT}\left(\left[s_{0}, E_{0}\right]\right)$.

Note that in both cases (1) and (2) the induction is valid because $E_{0}$ is the best node to start a tunnel going to its right. Indeed, starting at $E_{0}$ is cheaper, and no node closer to the sources can be reached (from the definition of $E_{0}$ ).

(3) $E_{1}$ on the right of $E_{0}$. Note that $E_{0}$ is a $(1,1)$ pseudo-source, while $E_{1}$ is a $(2,1)$ pseudo-source. Consider a tunnel ending in $\left[E_{1}+1, n\right]$. The situation gets more complicated than in the single source case, since $E_{1}$ (a $(2,1)$ node) is not the "best" possible node anymore. The only nodes that can beat $E_{1}$ are $(1,1)$ nodes, and $E_{0}$ is the rightmost one. Hence we can assume that such a tunnel is starting either at $E_{0}$ or at $E_{1}$. Indeed, we have two "best nodes". So to perform the induction we have to solve two subproblems: 
(3.1) the first subproblem on $\left[s_{0}, E_{1}-1\right]$, but under a condition on the location of the rightmost tunnel from $s_{1}$, i.e. $\operatorname{OPT}\left(\left[s_{0}, E_{1}-1\right] \mid\left(s_{1}, E_{1}\right)\right)$.

(3.2) the second subproblem in which we have two pseudo-sources $E_{0}$ of type $(1,1)$ and $E_{1}$ of type $(2,1)$. So we pay $\operatorname{OPT}\left(P\left((1,1),(2,1), l\left(E_{0}, E_{1}\right), l\left(E_{1}, E_{1}+1\right),\left[E_{1}+1, n\right]\right)\right)$.

To complete our algorithm we need to show how to compute the dynamic program tables inductively, i.e. to compute $\operatorname{OPT}\left(P\left((j, j),(j+1, j), l_{0}, l_{1},[u, v]\right)\right)$.

The two pseudo-sources tables. The induction is again on the two rightmost nodes $E_{0}, E_{1}$, with essentially the same cases as above, except case (1), which cannot occur since both pseudo-sources are now located outside the path.

(a) $E_{0}$ is on the right of $E_{1}$. Then $E_{0}$ is at distance $j+1$ from both sources and therefore any tunnel entering $\left[E_{0}+1, n\right]$ can be assumed to start at $E_{0}$. So the optimal solution is obtained by using $O P T\left(\left[s_{0}, E_{0}\right]\right)$ for the second subproblem and $O P T\left(P\left((j, j),(j+1, j), l_{0}, l_{1},\left[s_{0}, E_{0}-1\right]\right)\right)$.

(b) $E_{0}$ is on the left of $E_{1}$. The situation is similar to case (3). We can split the problem into two subproblems, the first one being a two pseudo-sources problem reduced to $\left[u, E_{1}-1\right]$ with a condition on the rightmost tunnel from $s_{0}$, and the second being a single source problem on $\left[E_{1}+1, n\right]$.

Correctness \& complexity. To complete the proof, we must explain how the above induction allows to compute all the tables inductively. Here are some explanations:

- First the induction is performed on the length of the path and when the tables for $[u, v]$ are computed, all the tables for strict subdipath of $[u, v]$ are known.

- Second, when filling the new tables, we compute the cost in a consistent way: we sum the cost of the first and second subproblems (found in already computed tables) with the cost of the tunnels that are removed, plus the hop count for traffic toward the removed node (either $E_{0}$ or $E_{1}$ ).

- As usual we keep only the best cost found when examining all the subcases 1,2,3.

- Finally, one may worry about the conditioning on the rightmost tunnel that appears in case (3). But fortunately this never leads to a condition on an unbounded number of tunnels, since in the induction those rightmost tunnels either disappear or stay.

To evaluate the complexity we use a pessimistic bound on the table size, $\operatorname{OPT}\left(P\left((j, j),(j+1, j), l_{0}, l_{1},[u, v]\right)\right)$. The values of $l_{0}, l_{1}$ are polynomial since they are in bijection with the pseudo-sources locations, $j \in[1, n]$. Since $[u, v]$ is either an end or a head segment we can store it in space $2 n$. Therefore we get size $\Theta\left(n^{4}\right)$ for the tables, and if we add the conditioning on the rightmost tunnel from the rightmost source we get $\Theta\left(n^{5}\right)$.

Finally, to improve the complexity we can use classical scaling technics to get space $\frac{\log n}{\varepsilon}{ }^{5}$ and approximation factor $1+\varepsilon$.

\section{Conclusions and Further Research}

In this paper we modeled a question raised by label minimization in GMPLS networks as a hypergraph layout problem. In the single commodity case we showed the problem to be closely related to well studied VPL problems. However, the optimization criteria (average hop count and average load) that appear in our problem are ones of the less studied. We observed that approximation results will immediately follow from extension of the results known for fixed depth hierarchical facility location (equivalently, bounded depth metric Steiner trees) to the average depth case. We also gave a general $\log n$-approximation that is universal (that is, it does not depend on the traffic), as well as hardness results.

In the multi-sources case, we presented a dynamic program on the path that is polynomial when the number of sources if fixed. So finding a polynomial algorithm in the general case on the path remains open; likely extensions of the dynamic program to the case of trees and bounded treewidth networks remains to be done. Last, we believe that more general approximation results can be given for low dimension Euclidean metric graphs using the classical Arora paradigm. 


\section{References}

1. Approximate Hierarchical Facility Location and Applications to the Shallow Steiner Tree and Range Assignment Problems. pages 211-222, 2006.

2. J.-C. Bermond, D. Coudert, J. Moulierac, S. Perennes, H. Rivano, I. Sau, and F. Solano Donado. MPLS label stacking on the line network. In Proceedings of IFIP Networking, Aachen, Germany, May 2009. Research Report INRIA RR-6803 accesible at http://hal.inria.fr/inria-00354267.

3. J.-C. Bermond, N. Marlin, D. Peleg, and S. Pérennes. Directed virtual path layouts in ATM networks. Theoretical Computer Science, 291(1):3-28, 2003.

4. M. Bern and P. Plassmann. The Steiner problem with edge lengths 1 and 2. Information Processing Letters, 32:171-176, 1989.

5. S. Bhatnagar, S. Ganguly, and B. Nath. Creating Multipoint-to-Point LSPs for traffic engineering. IEEE Commun. Mag., 43(1):95-100, Jan. 2005.

6. M. Charikar, C. Chekuri, T. Cheung, Z. Dai, A. Goel, S. Guha, and M. Li. Approximation algorithms for directed Steiner problems. In Proceedings of the 9th Annual ACM-SIAM Symposium on Discrete Algorithms (SODA), pages 192-200, 1998.

7. F. A. Chudak. Improved approximation algorithms for uncapacitated facility location. pages 180-194. Springer, 1998.

8. O. Gerstel, A. Wool, and S. Zaks. Optimal layouts on a chain ATM network. Discrete Applied Mathematics, 83:157-178, 1998.

9. M. X. Goemans, A. V. Goldberg, S. Plotkin, D. B. Shmoys, E. Tardos, and D. P. Williamson. Improved approximation algorithms for network design problems. In Proceedings of the 5th annual ACM-SIAM symposium on Discrete algorithms (SODA), pages 223-232, 1994.

10. S. Khuller and U. Vishkin. Biconnectivity approximations and graph carvings. Journal of the ACM, 41:214-235, 1994.

11. F. Ramos et al. IST-LASAGNE: Towards all-optical label swapping employing optical logic gates and optical flipflops. IEEE J. Sel. Areas Commun., 23(10):2993-3011, Oct. 2005.

12. R. Raz and S. Safra. A sub-constant error-probability low-degree test, and a sub-constant error-probability PCP characterization of NP. In Proceedings of the 29th annual ACM Symposium on Theory of Computing (STOC), pages 475-484, 1997.

13. H. Saito, Y. Miyao, and M. Yoshida. Traffic engineering using multiple MultiPoint-to-Point LSPs. In Proc. IEEE INFOCOM 2000, pages 894-901, 2000.

14. F. Solano, R. V. Caenegem, D. Colle, J. L. Marzo, M. Pickavet, R. Fabregat, and P. Demeester. All-optical label stacking: Easing the trade-offs between routing and architecture cost in all-optical packet switching. In IEEE Conference on Computer Communications (Infocom 08), pages 655-663, Phoenix, AZ, USA, Apr. 2008.

15. F. Solano, R. Fabregat, and J. Marzo. On optimal computation of MPLS label binding for MultiPoint-to-Point connections. IEEE Trans. Commun., 56(7):1056-1059, July 2007.

16. F. Solano and J. Moulierac. Routing in All-Optical Label Switched-based Networks with Small Label Spaces. In 13th Conference on Optical Network Design and Modeling (ONDM), Feb. 2009.

17. F. Solano, T. Stidsen, R. Fabregat, and J. Marzo. Label space reduction in MPLS networks: How much can one label do? IEEE/ACM Trans. Netw., Feb. 2009. 